\title{
Numerical simulations of a centrifuge model of caving
}

\author{
D Cumming-Potvin Australian Centre for Geomechanics, The University of Western Australia, Australia
}

J Wesseloo Australian Centre for Geomechanics, The University of Western Australia, Australia

ME Pierce Pierce Engineering, USA

T Garza-Cruz Itasca Consulting Group, USA

L Bouzeran Itasca Consulting Group, USA

SW Jacobsz University of Pretoria, South Africa

E Kearsley University of Pretoria, South Africa

\begin{abstract}
Validation and calibration of numerical models is vitally important, particularly in the field of cave mining where our ability to monitor the caving rock mass is limited. As part of a project investigating caving mechanics, physical models of caving were tested in a geotechnical centrifuge (Cumming-Potvin et al. 2016b). This paper describes numerical simulations of one of the centrifuge tests. Two approaches were used, so the relative strengths and weaknesses could be compared. The Itasca caving algorithm approach was implemented in FLAC3D and a bonded block model (BBM) approach implemented in 3DEC. The results showed that there was a good match with the physical model in some respects. Both numerical approaches were able to capture the discontinuity in the damage profile seen in the physical model, and the shape of the failed zones also matched the physical model well.

The match appeared to be better for the BBM approach. There is some uncertainty as to whether the mechanism of failure seen in the physical model was exhibited in the numerical models. The match between the numerical and physical models could have been improved via further calibration. However, this was outside the scope of this study. Overall, the results show that the numerical approaches used are suitable for practical use in the modelling of caving, particularly if they are suitably calibrated and/or validated using in situ monitoring data.
\end{abstract}

Keywords: numerical modelling, cave mining, physical modelling, centrifuge, fracture banding

\section{Introduction}

As part of a project investigating caving propagation mechanisms, a physical modelling program was conducted at the University of Pretoria Geotechnical Centrifuge Laboratory. The overall results of the project, focusing on the development of an extended conceptual model of caving, are given in Cumming-Potvin et al. (2018). This paper focuses on another aspect of the project.

The results of the physical modelling provided a unique opportunity for the testing, validation and improvement of numerical modelling approaches. Numerical modelling of one of the physical models was undertaken by staff from Itasca Consulting Group. The numerical models were performed before the materials testing of the physical modelling material was conducted, and a number of assumptions on the strength of the material had to be made at the time.

Comparing the results of the physical models with the numerical models highlights the different strengths and weaknesses of the approaches used, and reveals some interesting insights into the potential fracturing mechanisms. An in-depth validation and subsequent improvement of the modelling approaches is outside the scope of the current project. 


\section{$2 \quad$ Physical modelling}

The physical modelling involved using a centrifuge to accelerate a sample to $80 \mathrm{~g}$. By accelerating to many times the Earth's gravity, the test becomes representative of a cave many times the actual size of the sample, explained by the scaling law given in Hoek (1965). The samples were created from a weakly cemented sand and fly ash mixture. A quasi-random network of fully healed joints was cut into the sample in order to promote caving in a similar manner to the way joints promote caving in the rock mass in caving mines. The samples were housed in an aluminium frame, with thick glass panes in front of the sample. The tests were essentially two-dimensional, with an on-board DSLR camera allowing visual observations of the caving behaviour. The vertical stress was due to the self-weight of the sample (multiplied by the centrifuge's acceleration) and horizontal stress was supplied by water-filled bladders on either side of the sample. The undercut and draw of caved materials was simulated via five retractable pistons which sat under the sample. A schematic of the test design is given in Figure 1.

The way in which the frame restrained the sample along with the two-dimensionality of the test created conditions similar to plane-strain. The two-dimensionality inherently limited the test's ability to simulate cave propagation in the third dimension. However, it allowed valuable visual observations of the caving behaviour to be made.

More details on the physical modelling can be found in Cumming-Potvin et al. (2016a, b) and Cumming-Potvin (2018).

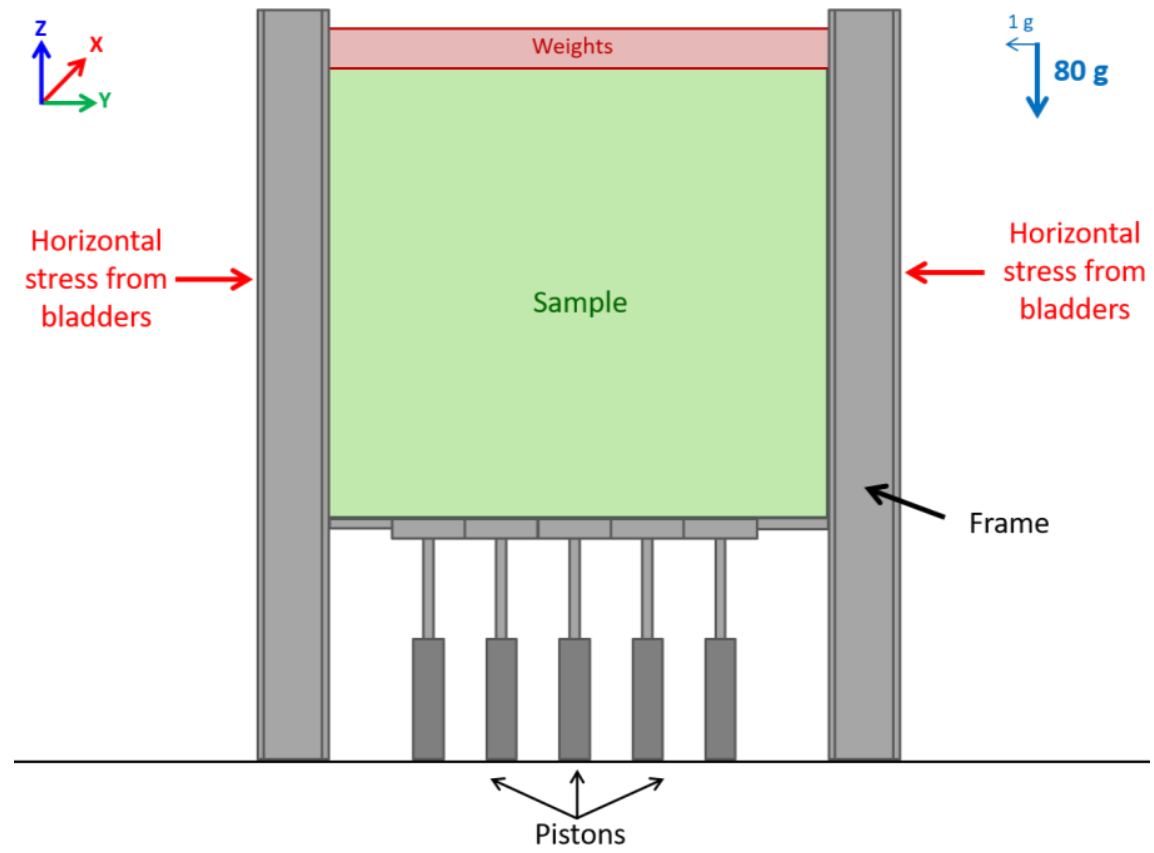

Figure 1 Schematic of centrifuge test design

\section{Approaches used}

Two different approaches were used: the Itasca caving algorithm approach and a bonded block model (BBM) approach.

\subsection{Itasca caving algorithm}

The Itasca caving algorithm, developed as part of the International Caving Study and Mass Mining Technology projects, implements the CaveHoek constitutive model in a continuum-based software, along with a set of functions to control draw. The CaveHoek constitutive model involves using strain-softening and strain-dependent material properties to represent caving behaviour. More details on the Itasca caving algorithm can be found in Board \& Pierce (2009). 


\subsection{Bonded block model}

The BBM approach involves representing the rock mass as a series of tetrahedral blocks with bonds at the contacts between blocks. In this modelling approach, the material behaviour is not explicitly specified. Instead, the properties of the bonds are altered to change the emergent macro material properties and behaviour. More detail on the BBM approach can be found in Garza-Cruz and Pierce (2014). The BBM models were implemented in the discontinuum code 3DEC (Itasca Consulting Group 2016a), and the Itasca caving algorithm models were implemented in the continuum code FLAC3D (Itasca Consulting Group 2016b).

\section{$4 \quad$ Numerical modelling inputs}

\subsection{Stress, dimensions and boundary conditions}

The same stress, dimensions and boundary conditions were used in both the 3DEC BBM models and FLAC3D Itasca caving algorithm models.

Stress: The stresses were initialised to be equal to the theoretical stress distribution from the physical models (Figure 2). The acceleration was also matched with the physical model with $80 \mathrm{~g}$ acceleration in the $Z$ direction and $1 \mathrm{~g}$ in the $\mathrm{Y}$ direction. The non-body forces were applied on the boundaries of the sample.

Dimensions: As 3DEC and FLAC3D are three-dimensional software packages, the dimensions of the samples and pistons could be represented exactly. The sample measured $500 \mathrm{~mm}$ in the $Y$ dimension, $450 \mathrm{~mm}$ in the $Z$ dimension, and $50 \mathrm{~mm}$ in the $X$ dimension.

Boundary conditions: The front and back of the sample were fixed in the $X$ direction but free to move in the $Y$ and $Z$ direction, while the sides of the sample were free only in the $Z$ direction. The top of the sample was free and the bottom of the sample completely fixed. Stresses were applied at the boundaries, including stress on the top of the sample to account for weights on top of the sample and stress on the side of the model which was applied by bladders in the physical model. The boundary conditions are shown in Figure 3 . Assuming free movement on the front and back of the sample is a simplification of the physical model, as there would be some friction on the glass surface to the front and the frame to the back (although a low-friction plastic sheet was used to reduce friction on the back of the sample).

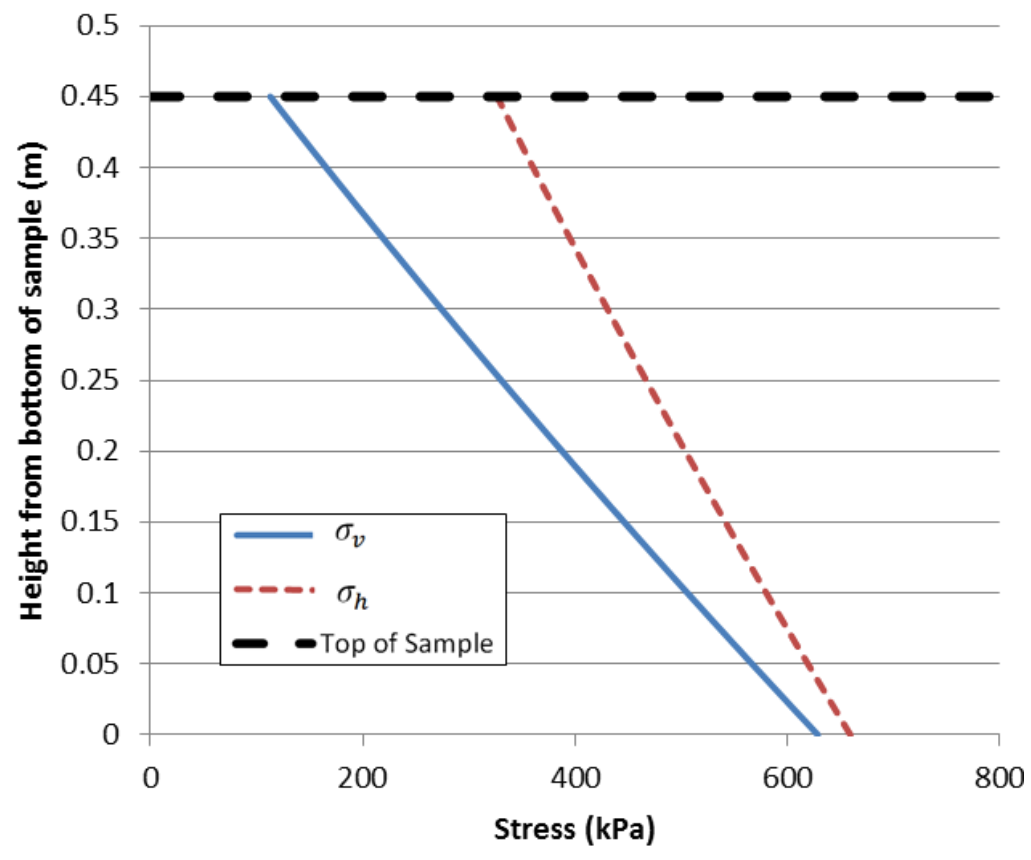

Figure 2 Stress distribution within sample 


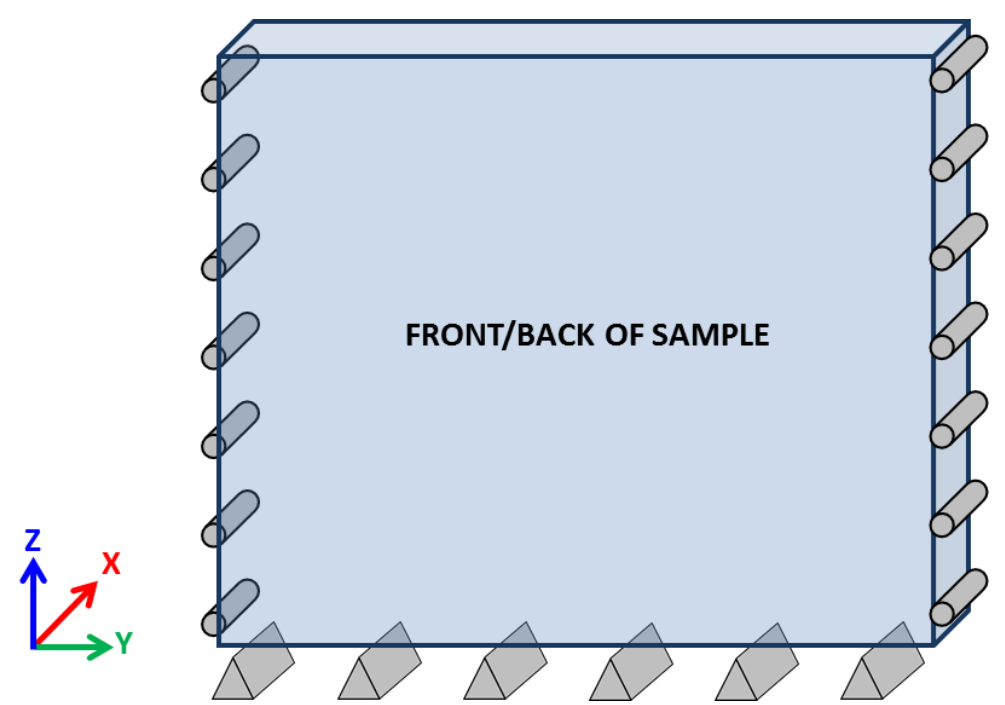

(a)

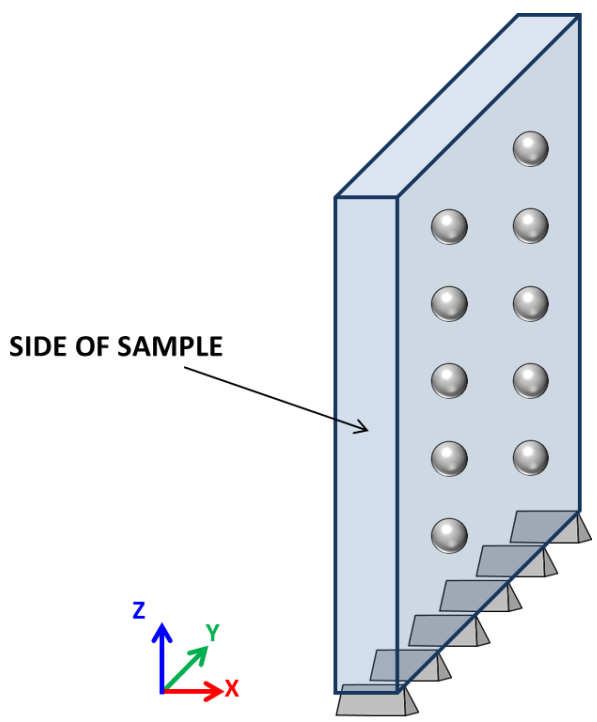

(b)

Figure 3 Boundary conditions; (a) Section view from front/back; and, (b) Section view (from side)

In both modelling approaches, the cave draw through piston retraction was implemented to represent the physical test, although this was done in different ways. The fundamental difference between the two approaches centres around the way the material behaviour is modelled.

\subsection{DEC - BBM model}

Elements: The elements were tetrahedral, with an edge length of $10 \mathrm{~mm}$ ( $2 \%$ of sample width and $20 \%$ of sample thickness).

Draw: The pistons were represented explicitly in the model and the data from the linear variable displacement transducers (LVDT) attached to the pistons was used so that the exact movement of the pistons could be replicated numerically.

Joints: The joints were modelled implicitly as part of the material. The joint pattern in the physical model was semi-random, and it was assumed that the randomly assigned variation in contact strength would result in a sufficient approximation of the joint pattern.

Material properties: The material properties of the physical model were unknown at the time the numerical models were created, and thus the properties had to be assumed. The same intact unconfined compressive strength (UCS) values were used for the 3DEC model as the FLAC3D model ( 1 and $0.75 \mathrm{MPa}$ ). Although these result in differing emergent rock mass strengths, this was deemed acceptable, as the purpose of the models was to compare failure mechanisms. In the BBM approach, the material is made up of a series of blocks which are bonded at their contacts. The micro-scale bond and contact strengths are assigned values such that the emergent macro-scale properties of the modelled material are equal to the assumed values. The emergent macro-scale properties are usually determined through the use of a numerical simulation of a UCS test. The process of assigning micro-scale properties requires the shape of the contact tensile strength distribution to be assumed. The material properties for the model are summarised in Table 1. The assumed distributions of contact tensile strength for both 3DEC models along with an example of the element contacts coloured by tensile strength for model 2 is given in Figure 4 . 
Table 1 Material properties: 3DEC - BBM models

\begin{tabular}{lll}
\hline & Model 1 & Model 2 \\
\hline Block properties & & \\
Young's modulus & $400 \mathrm{MPa}$ & $400 \mathrm{MPa}$ \\
Poisson's ratio & 0.25 & 0.25 \\
Density & $1,877 \mathrm{~kg} / \mathrm{m}^{3}$ & $1,877 \mathrm{~kg} / \mathrm{m}^{3}$ \\
Contact properties & & \\
Normal stiffness & $7.25 \mathrm{GNm} / \mathrm{m}^{2}$ & $7.25 \mathrm{GNm} / \mathrm{m}^{2}$ \\
Shear stiffness & $3.62 \mathrm{GNm} / \mathrm{m}^{2}$ & $3.62 \mathrm{GNm} / \mathrm{m}^{2}$ \\
Peak friction angle & $30^{\circ}$ & $30^{\circ}$ \\
Residual friction angle & $30^{\circ}$ & $30^{\circ}$ \\
Dilation friction angle & $30^{\circ}$ & $30^{\circ}$ \\
Peak tensile strength & Varied randomly based on & Varied randomly based on \\
Residual tensile strength & 0 & distribution in Figure 4 \\
Peak cohesive strength & $2.5 \times$ tensile strength & 0 \\
Residual cohesive strength & 0 & $2.5 \times$ tensile strength \\
Emergent rock properties & & 0 \\
UCS & $0.75 \mathrm{MPa}$ & $1 \mathrm{MPa}$ \\
Young's modulus & $325 \mathrm{MPa}$ & $325 \mathrm{MPa}$ \\
\hline
\end{tabular}

The failure envelopes of the $10^{\text {th }}, 50^{\text {th }}$ and $90^{\text {th }}$ percentiles of the tensile strength distribution are shown in Figure 5. The tensile strength distribution is shown in pink for reference.
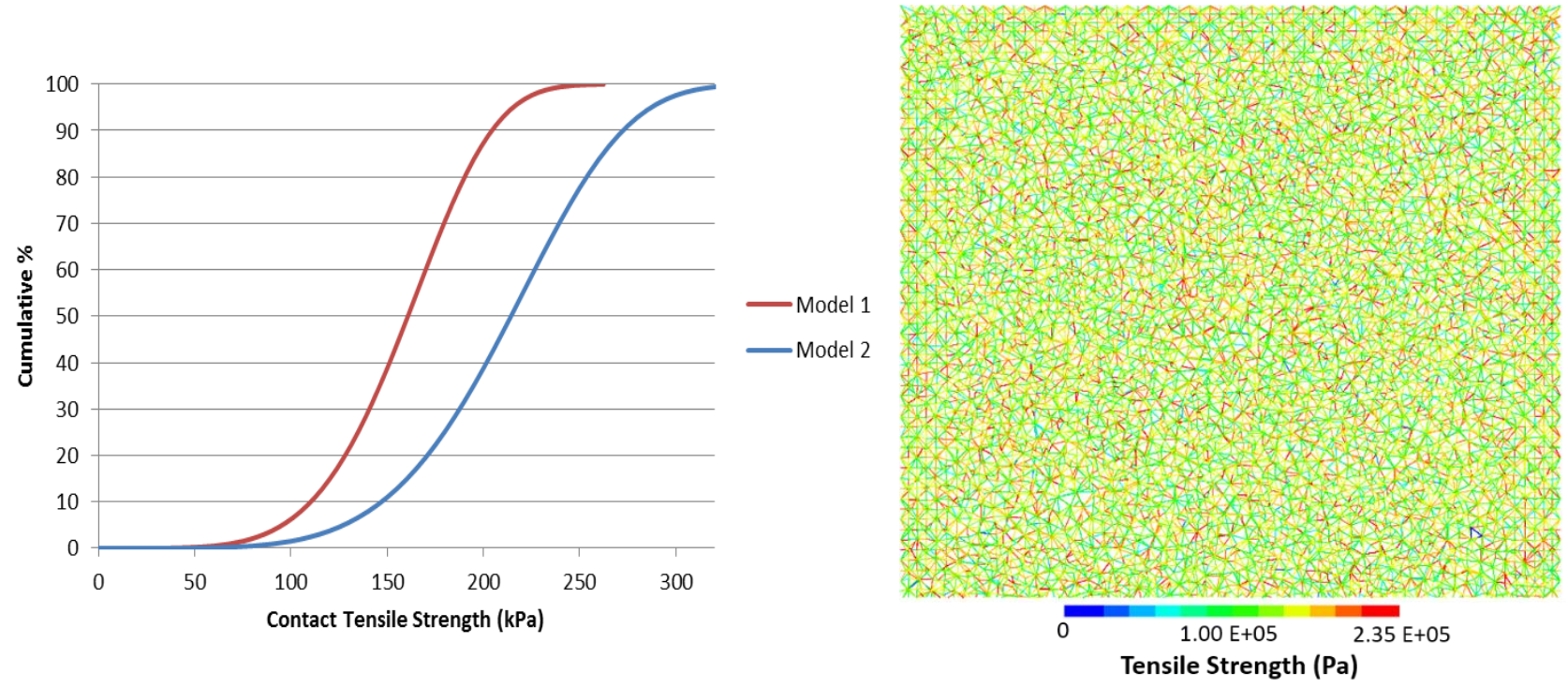

Figure 4 (a) Distribution of contact tensile strength for both models; and, (b) Element contacts coloured by tensile strength (model 2) 


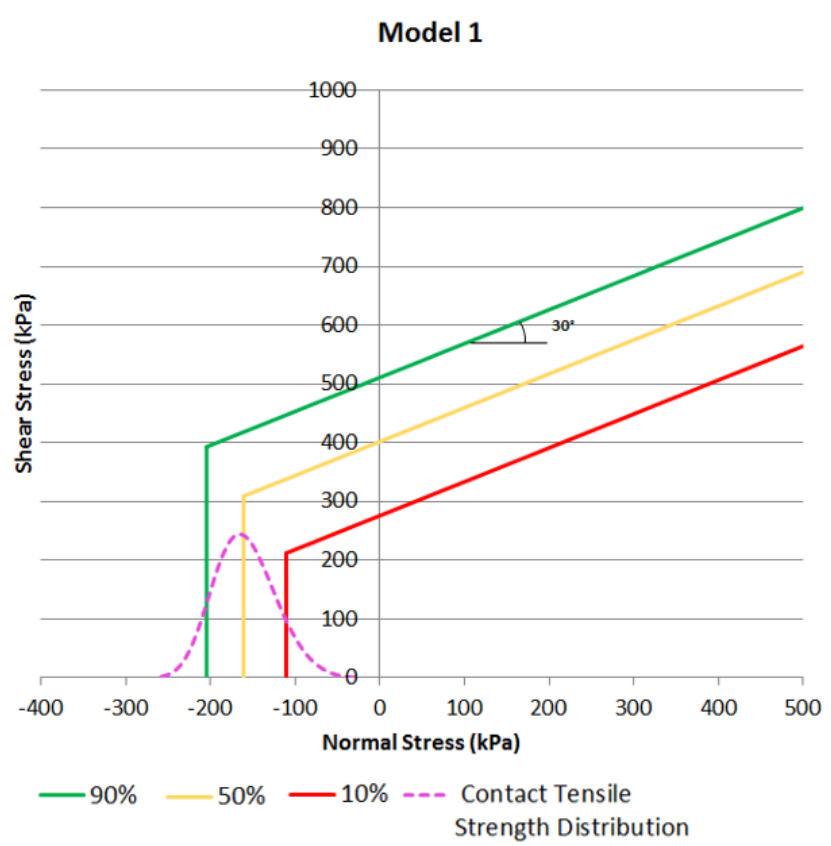

(a)

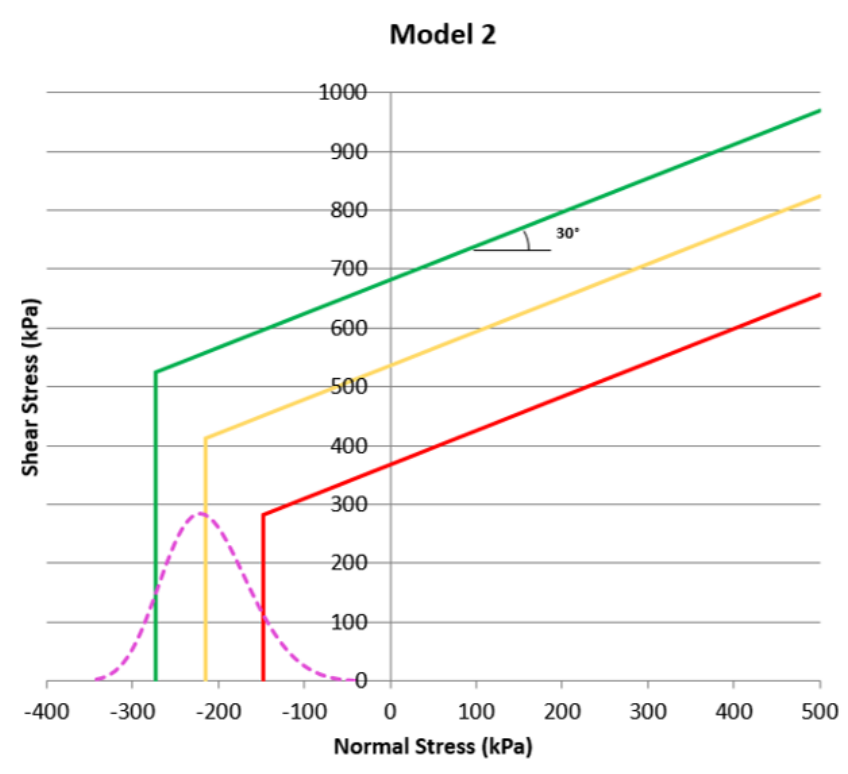

(b)

Figure 5 Failure envelopes; (a) Model $1-0.75 \mathrm{MPa}$; and, (b) Model $2-1 \mathrm{MPa}$

\subsection{FLAC3D - Itasca caving algorithm model}

Draw: A low velocity was applied at the bottom of the sample at the position of each piston to simulate the movement of each piston. The magnitudes of the velocities applied were scaled to replicate the relative displacement of each piston measured in the physical model. The model geometry and simulated pistons are illustrated in Figure 6.

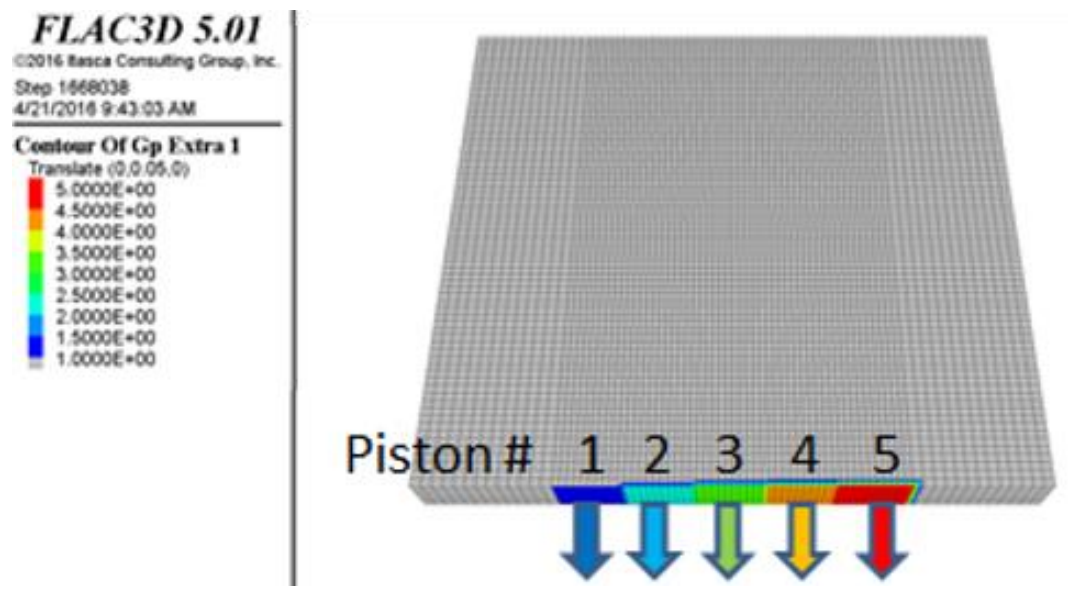

Figure 6 FLAC3D model geometry and pistons

Joints: The joints were modelled implicitly as part of the material. They were taken into account through the use of a Geological Strength Index (GSI) of 90. The sample strength is uniform throughout the sample, which is not the case in the BBM model. 
Material properties: Two different assumed intact UCS values were tested. At the time of the numerical modelling, only the wet density of the sample was known, and this value was used. The values of GSI, $m_{i}$, intact Young's Modulus, Poisson's ratio, critical plastic shear strain, residual cohesion and residual friction angle were assumed based on experience. The tensile strength of the material was assumed to be approximately $3 \%$ of the intact UCS. The values used for the two FLAC3D models are given in Table 2 .

Table 2 Input parameters for Itasca caving algorithm - FLAC3D models

\begin{tabular}{lll}
\hline & Model 3 & Model 4 \\
\hline UCS ${ }_{i}$ & $0.75 \mathrm{MPa}$ & $1 \mathrm{MPa}$ \\
GSI & 90 & 90 \\
$\mathrm{~m}_{\mathrm{i}}$ & 15 & 15 \\
Intact Young's modulus & $325 \mathrm{MPa}$ & $325 \mathrm{MPa}$ \\
Poisson's ratio & 0.25 & 0.25 \\
Density & $1,877 \mathrm{~kg} / \mathrm{m}^{3}$ & $1,877 \mathrm{~kg} / \mathrm{m}^{3}$ \\
Tensile strength & $24 \mathrm{kPa}$ & $31 \mathrm{kPa}$ \\
UCS & \\
Critical plastic shear strain & $2.8 \%$ & $611 \mathrm{kPa}$ \\
\hline
\end{tabular}

\section{$5 \quad$ Results}

\subsection{DEC - BBM model}

The results of the 3DEC BBM models are shown in Figures 7 and 8 . The shape of the caved zone (vertical displacement of greater than 0.012) in both BBM models matches that of the physical model relatively well. The lateral extents of the failed zone match the physical model particularly well. The vertical extents of the caved zones are somewhat smaller than those seen in the physical model and may be due to the high UCS value assumed in the numerical model. The numerical model indicates more small-scale fracturing when compared with the larger fractures seen in the physical model. Although, it is possible that there are smaller micro-scale fractures that are not visible in the photos. These smaller scale fractures in the numerical model appear to form in a 'fracture zone' which is parallel to the cave surface. Interestingly, the small fractures themselves appear to be oriented primarily in an orientation perpendicular to the cave surface. This contrasts with the larger fractures parallel to the cave seen in the physical model.

The 'fracture zones' are at times alternated with zones with less damage, creating a discontinuous damage profile similar to that seen in the physical models. This is illustrated in Figure 9. The cave also appears to progress vertically through 'jumps' to successive damage zones in a manner similar to the physical model. The results of model 2 ( $1 \mathrm{MPa}$ strength) show a similar caving behaviour to that of model $1(0.75 \mathrm{MPa})$, albeit with slightly smaller failure zones. The model indicated that a large portion of the fractures created were nucleated in shear, and subsequently propagate in tension. While the mechanism of fracture initiation in the physical models could not be determined with certainty, there were indications that the fractures formed and propagated in extension. 
PHYSICAL MODEL
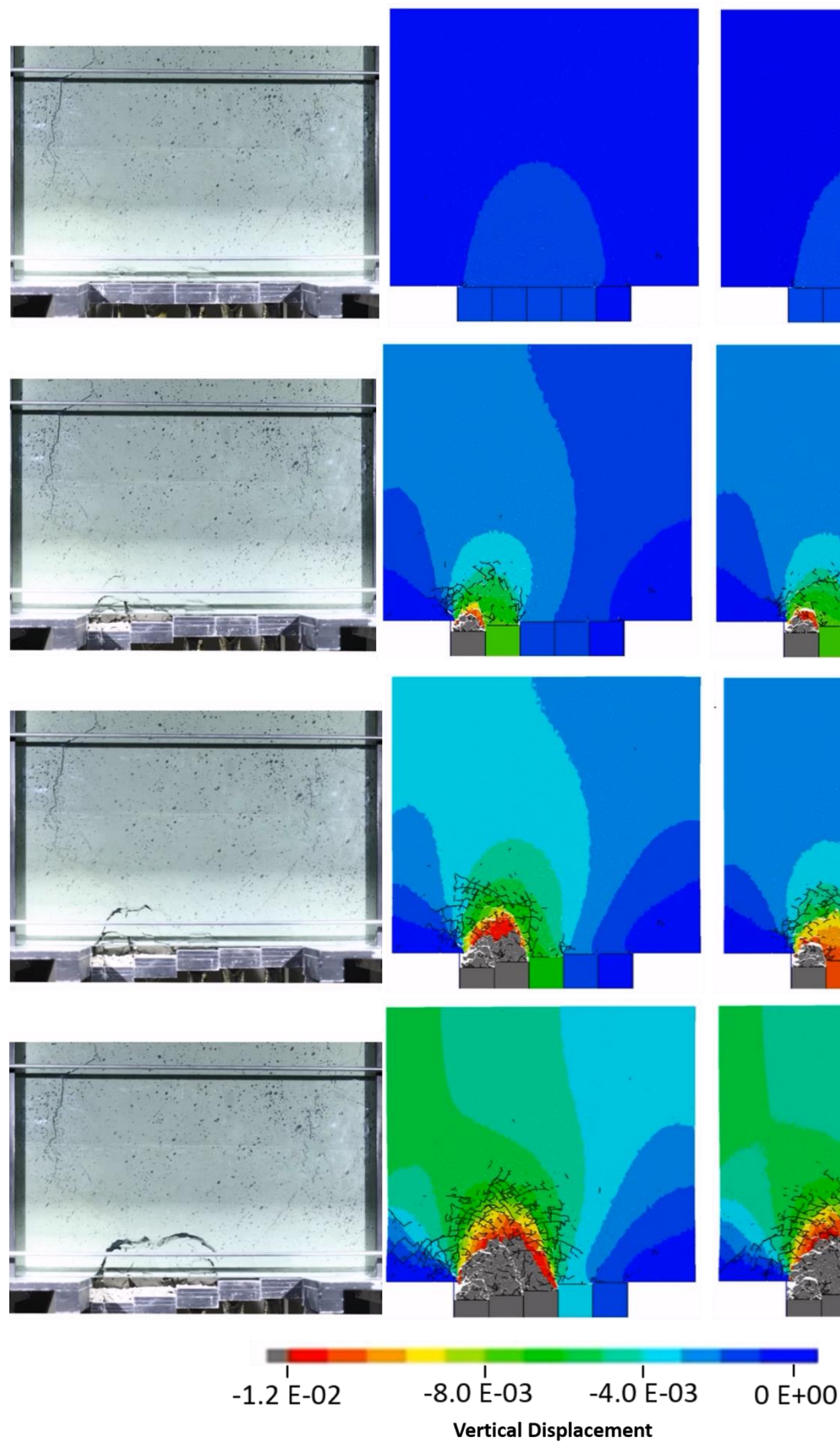

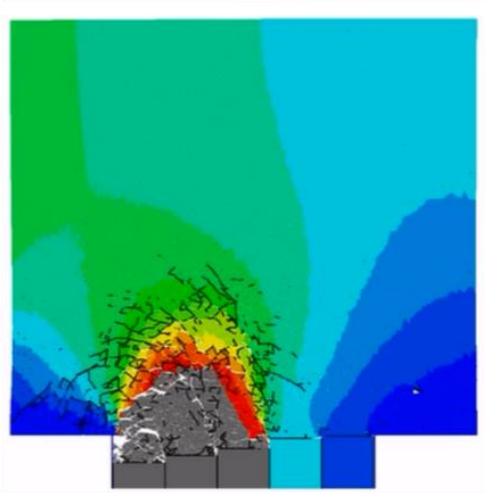

$\mathrm{BBM}-1 \mathrm{MPa}$
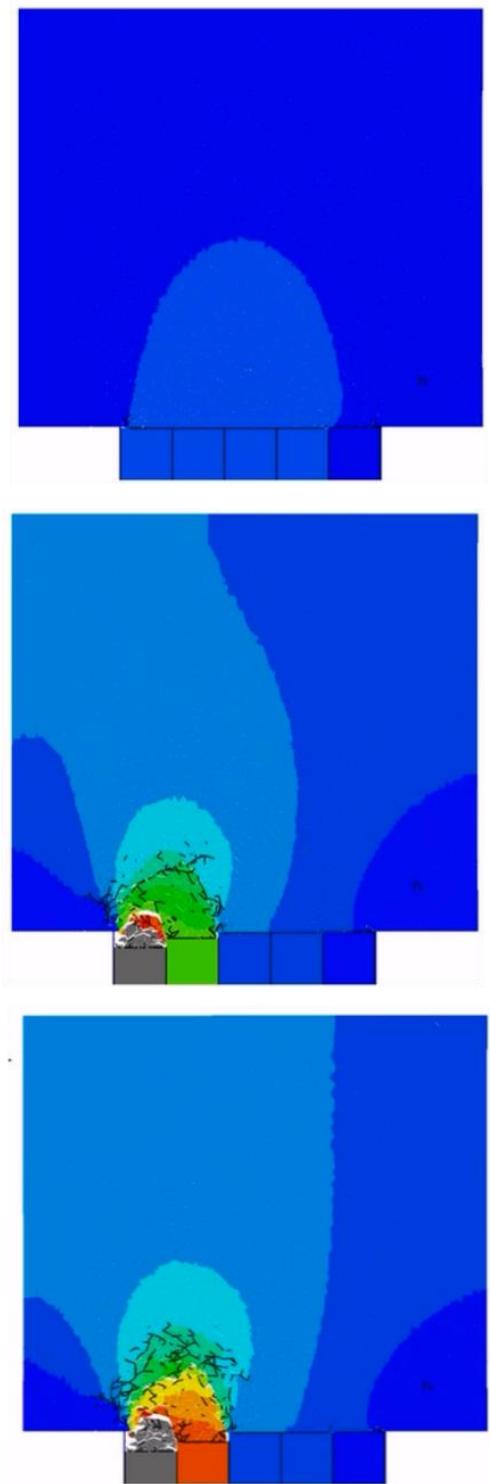

$+00$

Figure 7 Vertical displacement results of 3DEC BBM models showing contours of vertical displacement: part 1. Note: physical and numerical model results not to the same scale 
PHYSICAL MODEL
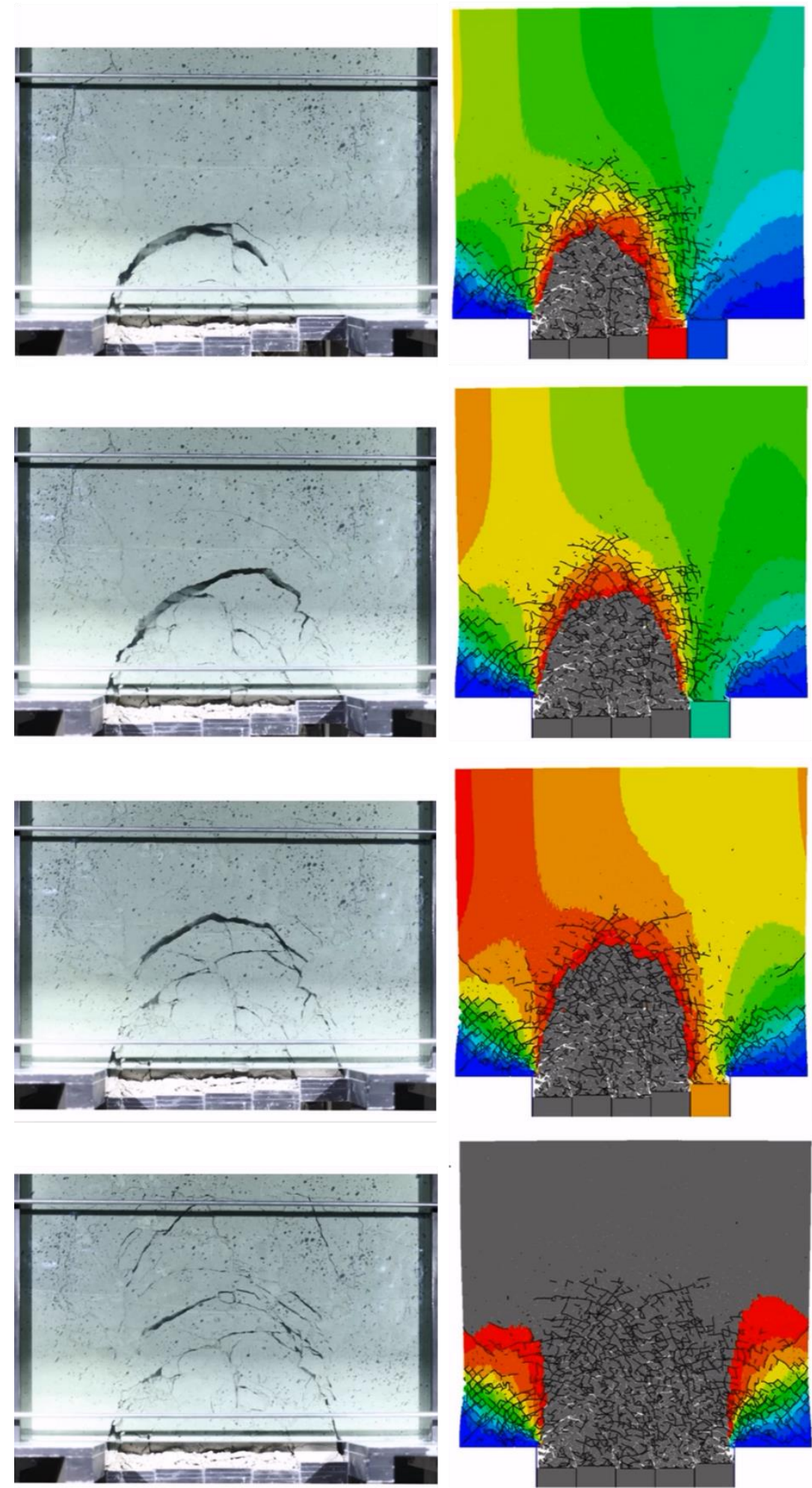

$\mathrm{BBM}-1 \mathrm{MPa}$
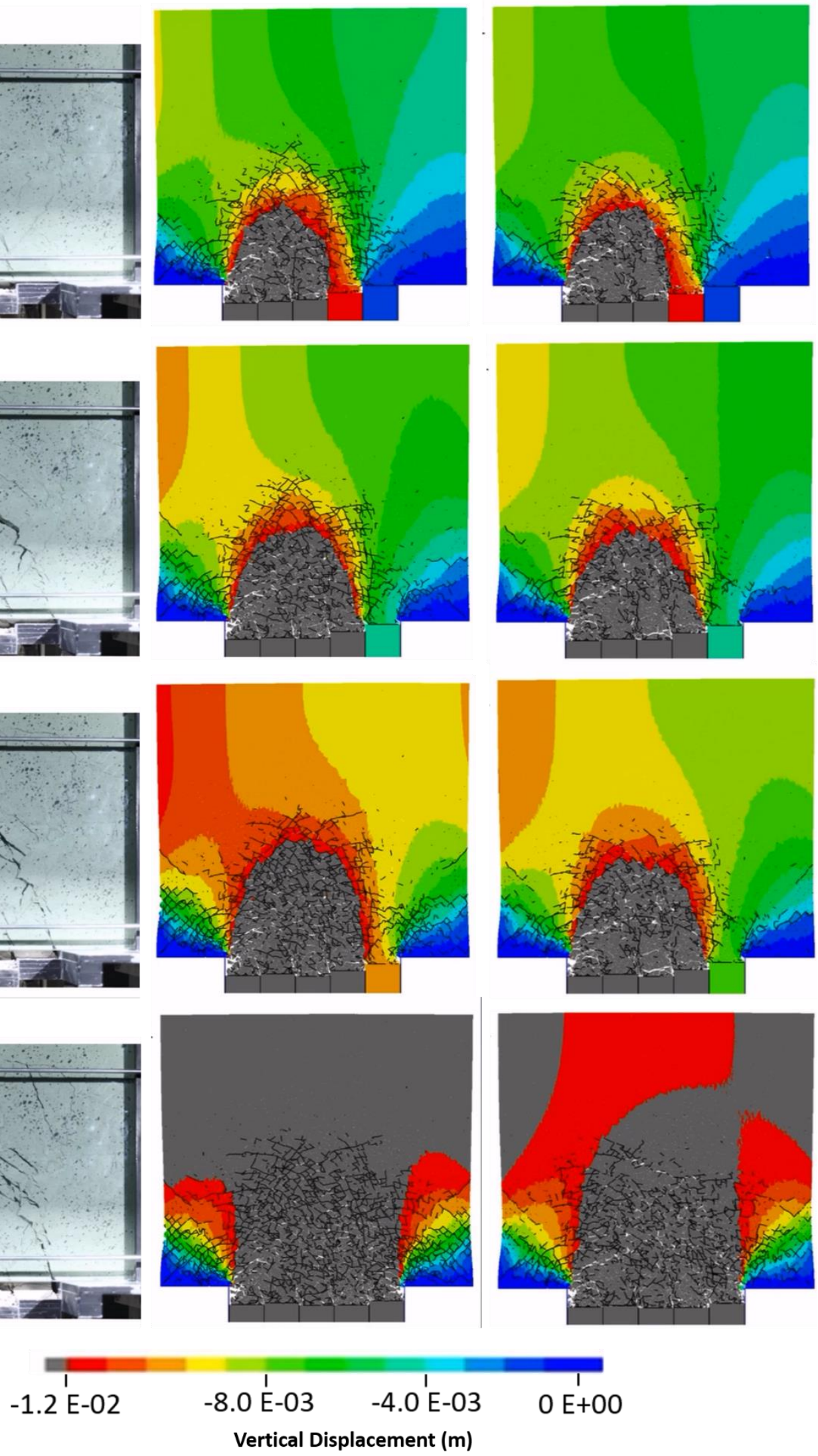

Figure 8 Vertical displacement results of 3DEC BBM models: part 2. Note: physical and numerical model results not to the same scale 


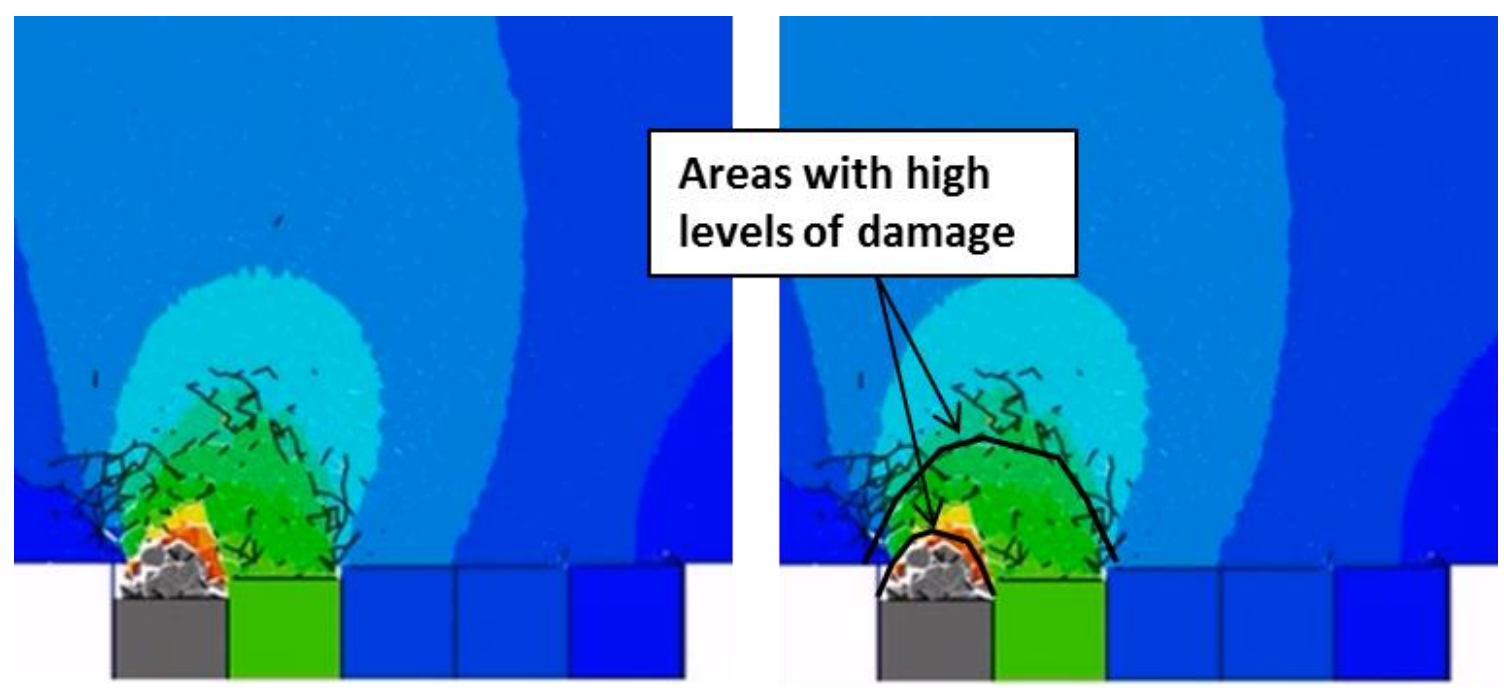

Figure 9 Example of discontinuous damage in 3DEC model

\subsection{FLAC3D - Itasca caving algorithm model}

Figure 10 gives the vertical displacement results of the FLAC3D models at different stages of cave propagation for the 0.75 and $1 \mathrm{MPa}$ UCS models respectively. Based on visual observations, it appears that the geometry of the cave in the $0.75 \mathrm{MPa}$ model matches better with the physical model than the $1 \mathrm{MPa}$ model. However, neither matches as well as the results of the 3DEC models.

In both the 0.75 and $1 \mathrm{MPa}$ FLAC3D models, the cave appears to stall towards the end of the test, which was not the case in the physical model. This may be attributed to the assumed value of UCS used being higher than that of the physical model. Limited discontinuity is seen in the vertical displacement results which may be attributed to the continuum code modelling approach not allowing true discontinuous behaviour.

Interestingly, the plastic shear strain results (shown in Figure 11) show shear localisation. Distinct shear bands form and propagate ahead of the cave. The shear bands form progressively, with multiple bands propagating simultaneously. The vertical extent of the shear bands is significantly larger than the parallel fractures seen in the physical model. This result suggests that the caving algorithm model may be over-predicting the height of the fractured zone. This over-prediction cannot be regarded as indicative of the accuracy of the modelling process, as the match may be improved by using improved material parameters. 
PHYSICAL MODEL
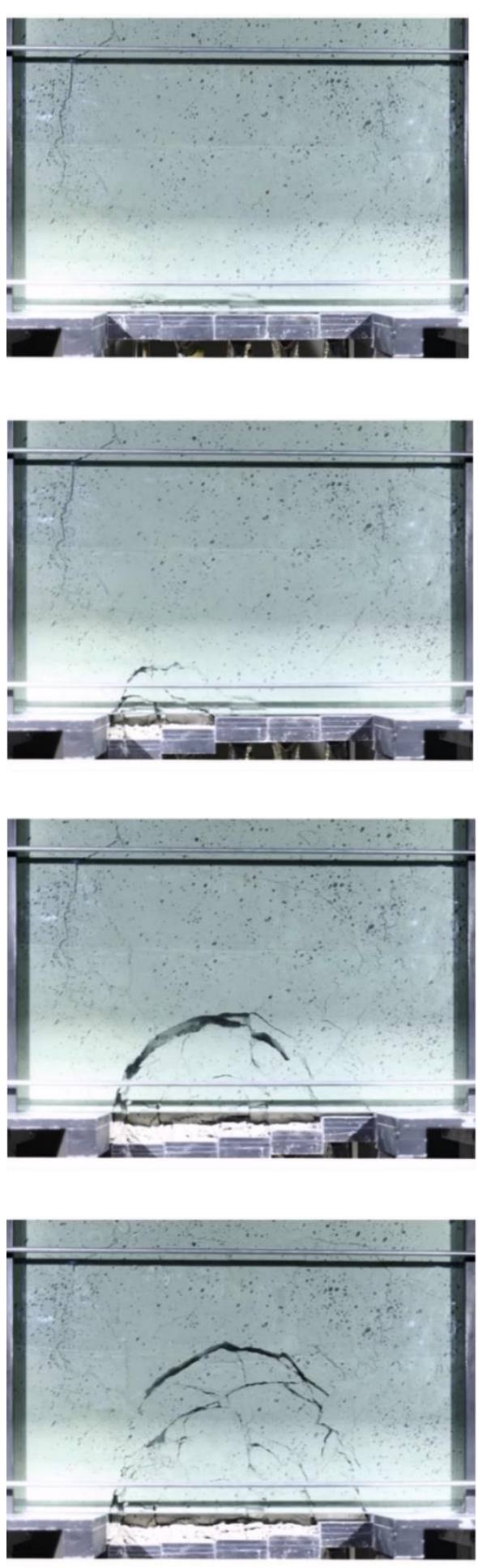

FLAC3D - $750 \mathrm{kPa}$
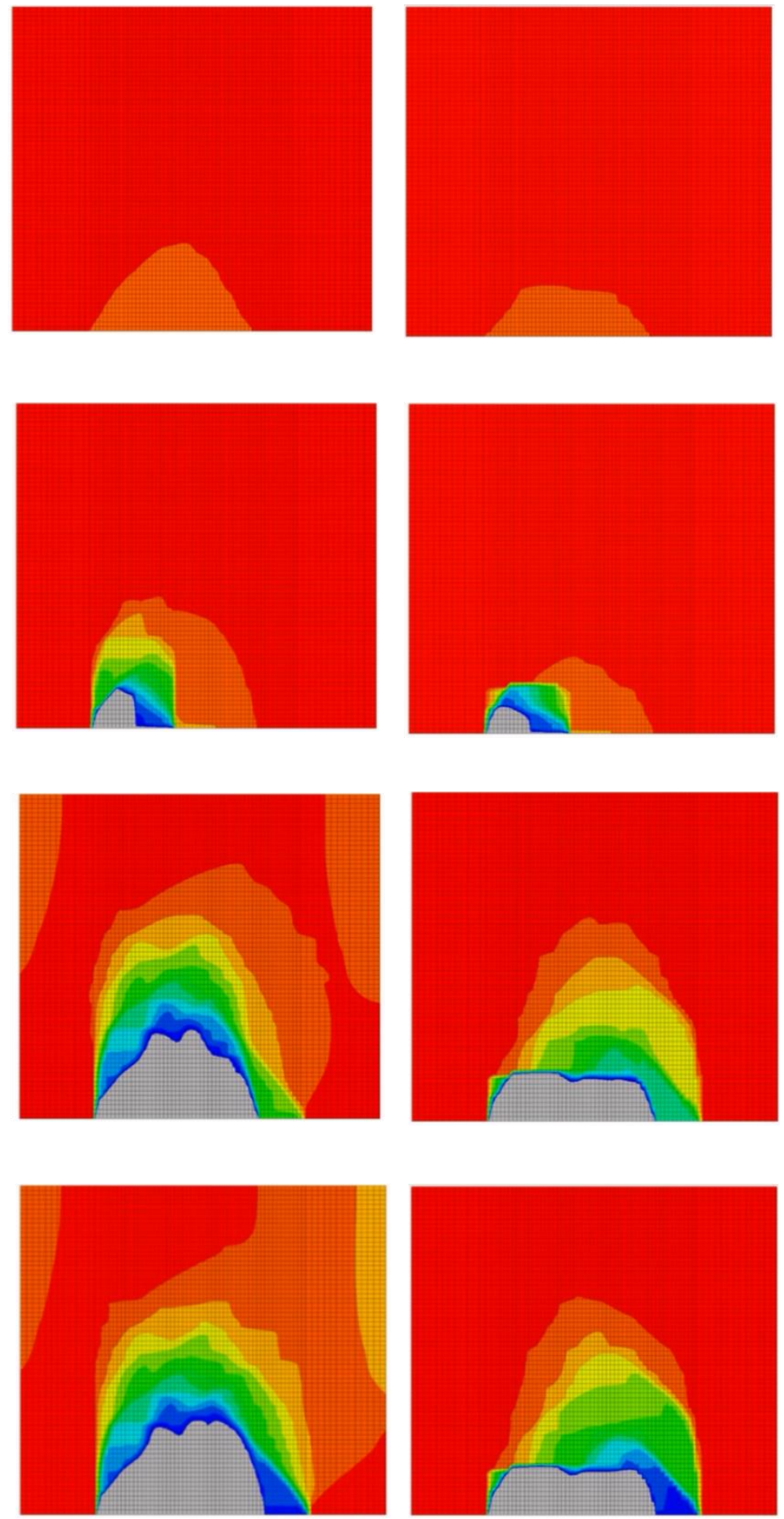

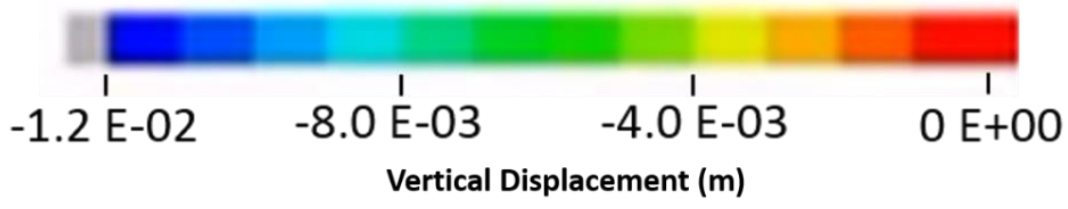

Figure 10 Vertical displacement - FLAC3D caving algorithm models. Note: physical and numerical model results not to the same scale 


\section{FLAC3D - $750 \mathrm{kPa}$}

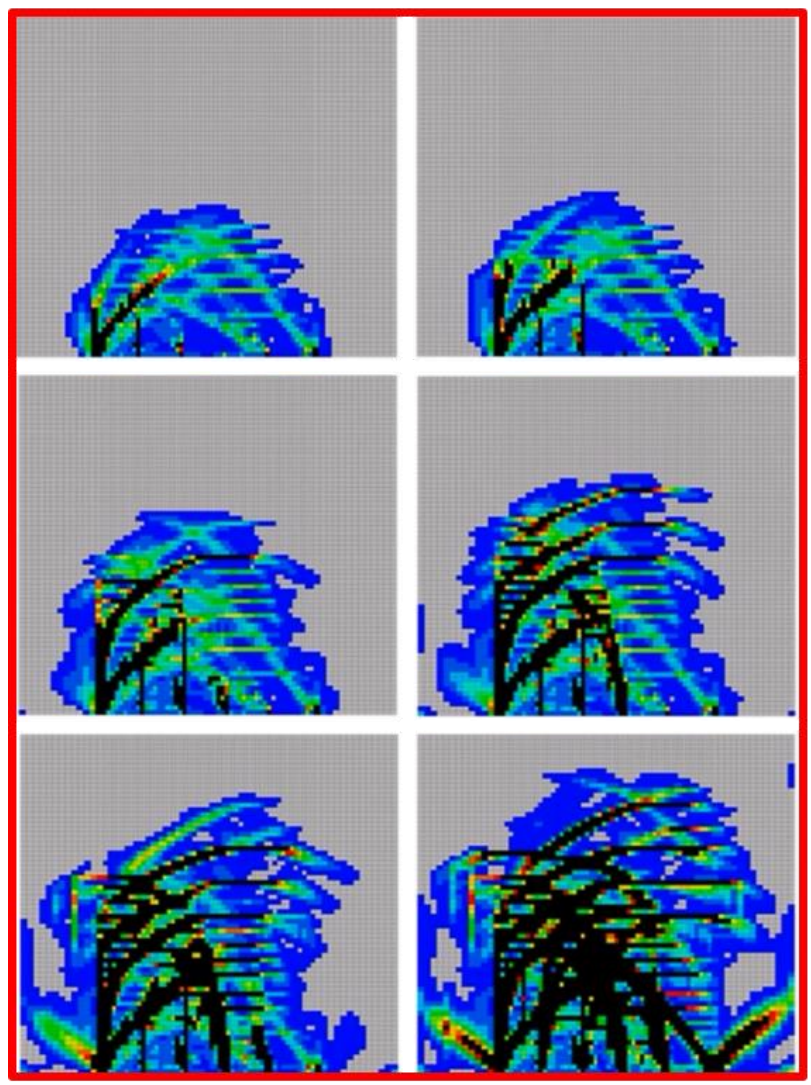

FLAC3D - $1 \mathrm{MPa}$

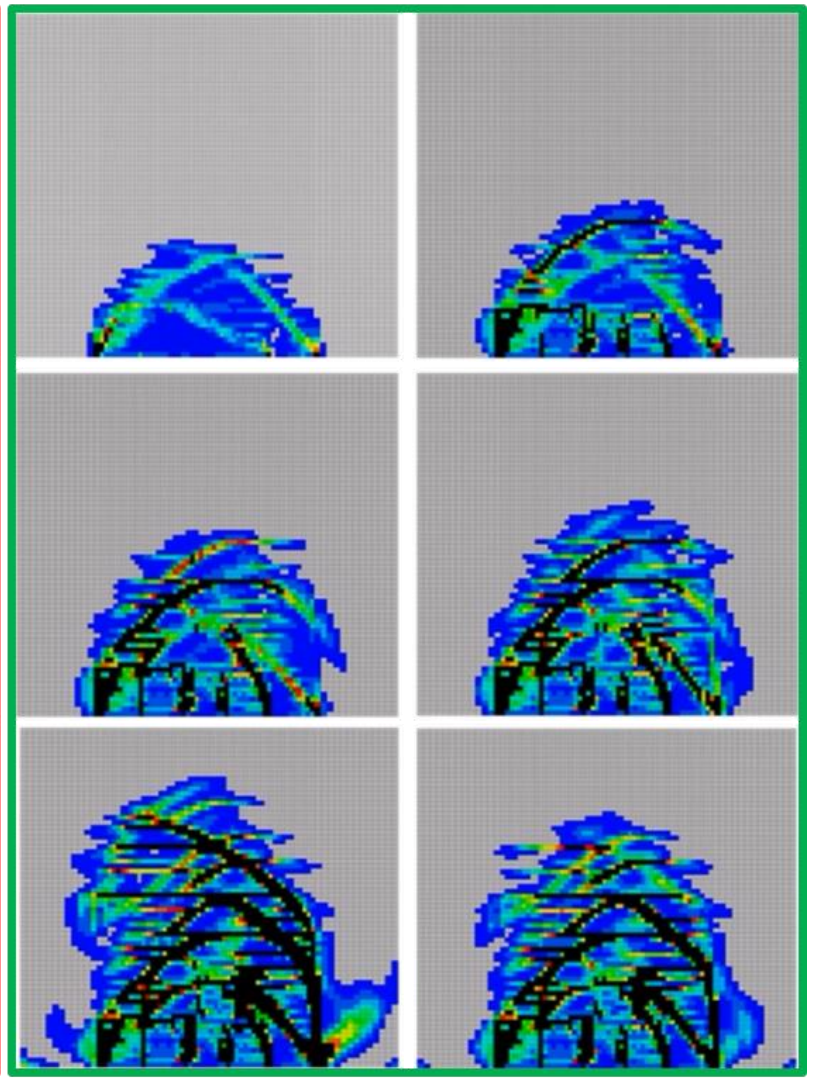

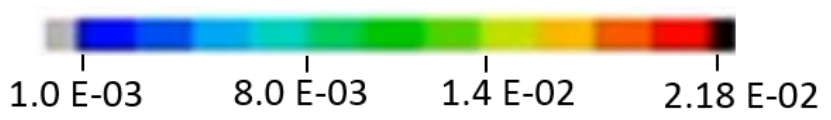

Figure 11 Plastic shear strain - FLAC3D caving algorithm model 3 (left); and, model 4 (right)

\section{Discussion}

Both numerical approaches showed strengths and weaknesses when simulating the physical model. In the 3DEC BBM models, the fractures tended to concentrate in bands, creating discontinuous damage zones similar to the parallel fractures from the physical model. The cave also appeared to progress through 'jumps' to the next successive damage zone. The models were also able to recreate the cave shape relatively well, in particular the lateral extents of the cave.

The size and orientations of the fractures created did not match as well with the physical model. The BBM models indicated smaller scale fracturing oriented primarily perpendicular to the cave back (concentrated into damage bands parallel to the cave back).

The cave geometry in the FLAC3D Itasca caving algorithm models did not match the physical model as well as the BBM. The caving process was more continuous, attributed to the bias towards continuum behaviour implicit in the approach. The models do, however, capture some of the discontinuous nature of the failure process from the physical models through the shear bands seen in the plastic shear strain plot. The shear bands formed simultaneously ahead of the cave in a manner similar to the physical model. While the FLAC3D software cannot explicitly model the formation of fractures, localised shear damage zones emerge in the model.

An important aspect of the comparison between the physical and numerical models is in the underlying failure mechanisms. The material models used in the FLAC3D model are based on Mohr-Coulomb/ Hoek-Brown failure criteria. As such, they cannot represent an extensional failure mechanism as described 
by Stacey (1981) and Wesseloo and Stacey (2016) (i.e. one in which a tension crack forms parallel to the major principal stress in a three-dimensional compressional stress environment). The failure mode seen in the Itasca caving algorithm FLAC3D model is more reminiscent of the shear bands formed in weak, low-dilatancy materials described by Barton (1993) and Crook et al. (2003) (Figure 12).

Although BBMs are capable of simulating tension crack initiation in a globally compressive environment, inspection of the failure mode of the element contacts in the BBM model revealed that the majority initiated in shear and propagated in tension. The fracture pattern observed resembles the fracture pattern of families of shear cracks creating spiral rupture surfaces seen in numerical models of a circular shaft by Lisjak et al. (2014). The fractures tend to develop at an angle of $45^{\circ}$ to the maximum principal stress. The similarities between the two fracturing patterns are illustrated in Figure 13.

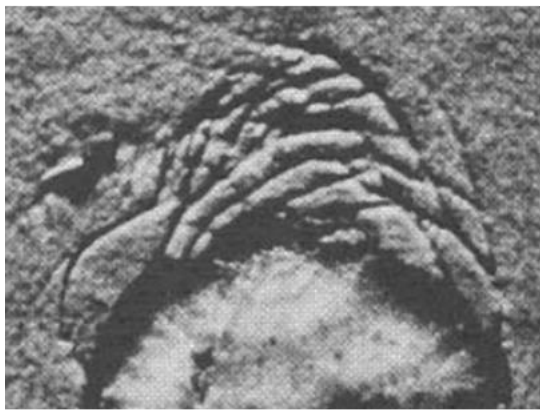

(a)

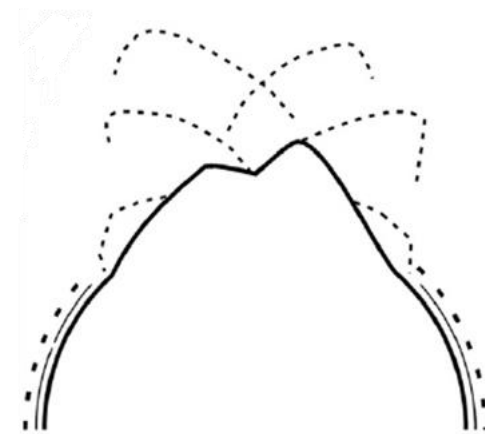

(b)

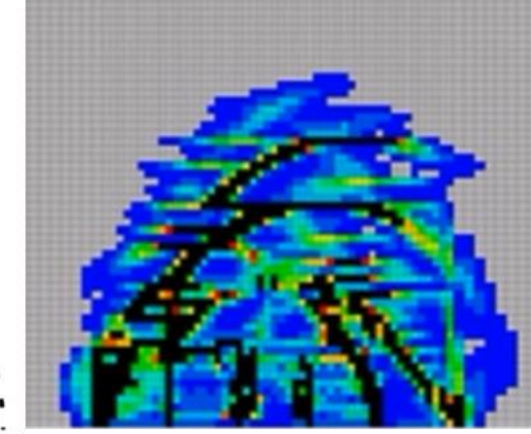

(c)

Figure 12 (a) Failure zones from boreholes in sandstone (Addis et al. 1990); (b) Shear bands from borehole breakout in black shale (Meier et al. 2013); and, (c) Plastic shear strain from Itasca caving algorithm FLAC3D model

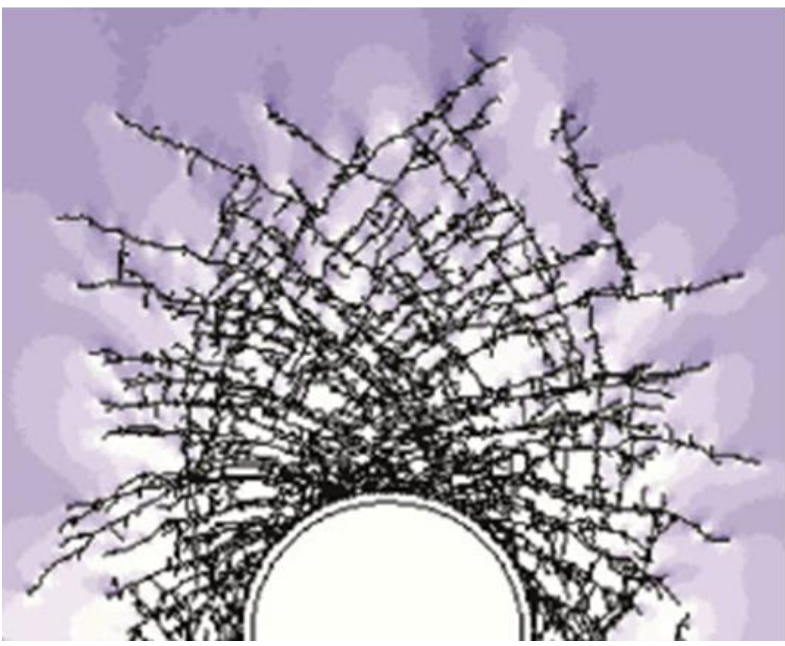

(a)

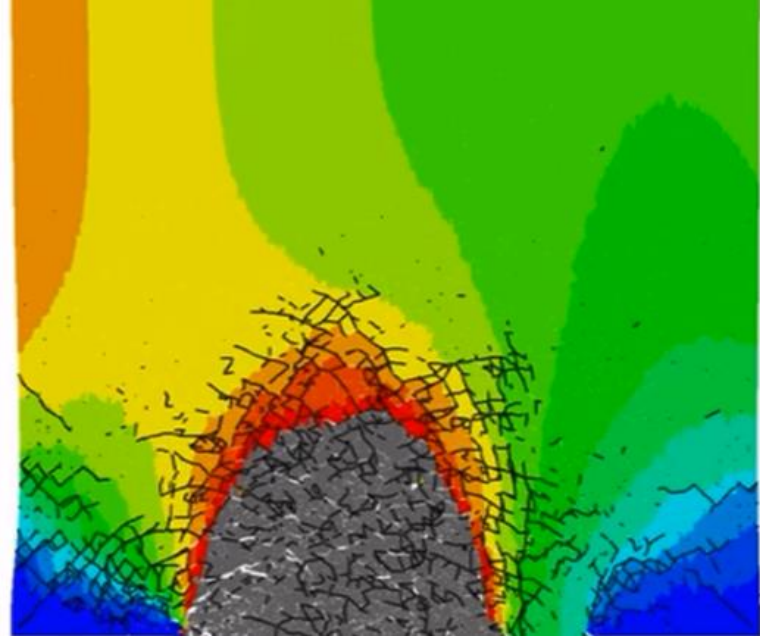

(b)

Figure 13 (a) Fracturing pattern around circular shaft (Lisjak et al. 2014); and, (b) Z displacement and fracturing results from $3 D E C B B M$ model

In contrast with this, the initiation and propagation mechanism of fracturing in the physical models appeared to be extension. The results indicated that the direction of movement was perpendicular to the fracture orientations. Additionally, the fracturing pattern observed in the physical models was similar to the axial splitting in strong, brittle materials described by Crook et al. (2003). This mode of borehole breakout (illustrated in Figure 14) occurs through extensional fracturing. Unfortunately, the frequency of photographs taken during the physical modelling was not fast enough to determine the fracturing mechanism with complete certainty. 

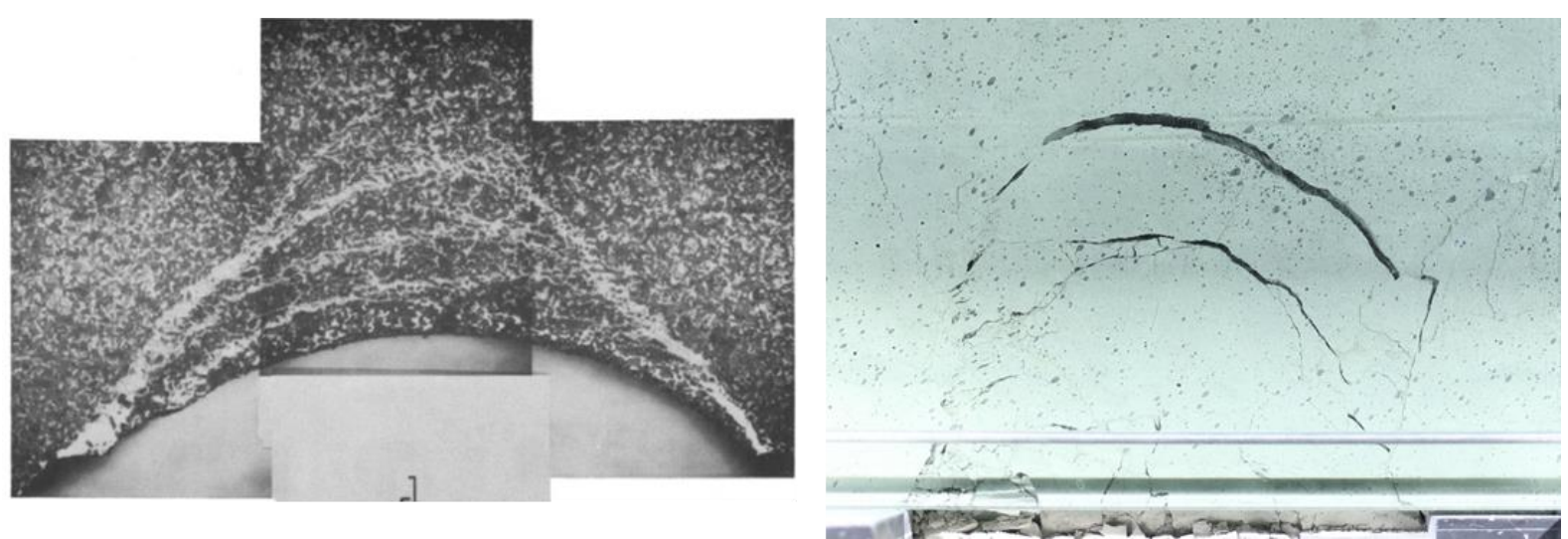

Figure 14 (a) Axial splitting through extensional failure (after Ewy \& Cook 1990); and, (b) Parallel fractures from physical modelling

The fracturing patterns of the shear bands in the continuum model and the fracture pattern emerging in the BBM model are similar to the patterns observed for known shear failure mechanisms. The fracturing pattern observed in the physical model is more akin to that from known extensional fracturing. From the differences in the fracture patterns observed (illustrated in Figure 15), we can conclude that the numerical models may not be fully capturing the mechanism seen in the physical models and that the material models could be further refined.

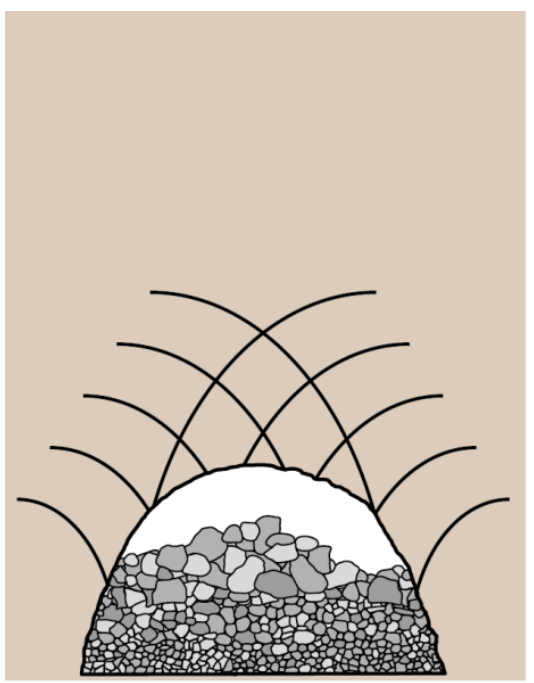

3DEC - BBM

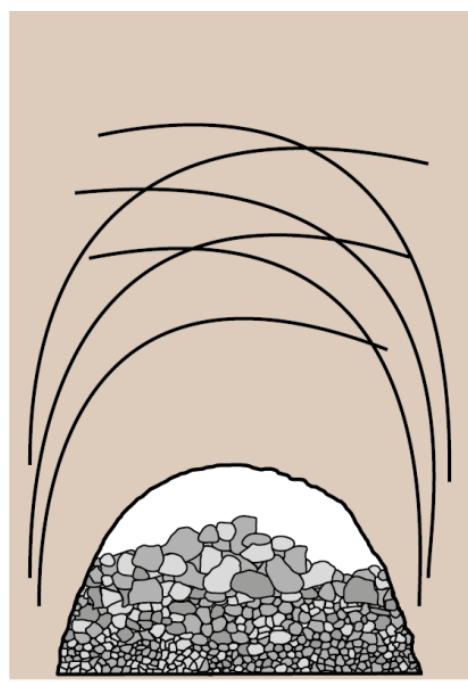

FLAC3D - Itasca

Caving Algorithm

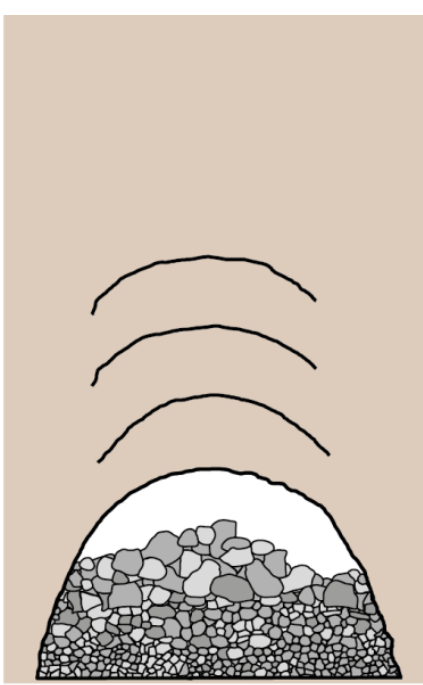

Physical Model

Figure 15 Schematic of observed fracture patterns

It is important to note that adjusting or calibrating the model to improve the match between the numerical and physical models was outside the scope of the project. It is possible that some of the limitations of the model could be overcome with adjustment of the modelling approach, input parameters and material model used.

\section{Conclusion}

The results of the numerical modelling provided a good match with the physical models in some respects. The discontinuity of the damage profile was captured by both the Itasca caving algorithm and BBM approaches, with the BBM showing the better match. The shape of the cave zones evolved also match the physical models relatively well. While the size of the failure zones did not match particularly well, this could likely be rectified with the use of a material strength which better matches that of the physical modelling material (rather than relying on assumptions). These results show that the models are suitable for practical 
use in the modelling of caving, particularly if the models are calibrated or validated using in situ monitoring data. There is, however, some uncertainty as to whether the mechanisms shown in the numerical models match those seen in the physical models. Further calibration of the numerical models with respect to the mechanism, while outside of the scope of this study, could improve the match with the physical models.

This study gives an example of how numerical models of caving can be validated against physical models tested in a geotechnical centrifuge. This process is of utmost importance, as it allows us to better understand the strengths and limitations of different modelling approaches, and gives confidence in the results from numerical models.

\section{Acknowledgement}

The authors thank the staff at the University of Pretoria Geotechnical Centrifuge Laboratory for their efforts in the centrifuge modelling project referenced in this paper.

\section{References}

Addis, MA, Barton, N \& Bandis, SC 1990, 'Laboratory studies on the stability of vertical and deviated boreholes', Proceedings of the 65th Annual Technical Conference and Exhibition of the Society of Petroleum Engineers, Society of Petroleum Engineers, Richardson, pp. 19-30.

Barton, N 1993, 'Physical and discrete element models of excavation and failure in jointed rock', Assessment and Prevention of Failure Phenomena in Rock Engineering, A.A. Balkema, Rotterdam, pp. 35-46.

Board, M \& Pierce, ME 2009, 'A review of Rrecent experience in modeling of caving', Proceedings of the International Workshop on Numerical Modeling for Underground Mine Excavation Design, National Institute for Occupational Safety and Health, Washington, D.C., pp. 19-28.

Crook, T, Willson, S, Yu, JG \& Owen, R 2003, 'Computational modelling of the localized deformation associated with borehole breakout in quasi-brittle materials', Journal of Petroleum Science and Engineering, vol. 38, pp. 177-186.

Cumming-Potvin, D 2018, An Extended Conceptual Model of Caving Mechanics, PhD thesis, The University of Western Australia, Perth.

Cumming-Potvin, D, Wesseloo, J, Jacobsz, SW \& Kearsley, EP 2016a, 'Fracture banding in caving mines', The Journal of the Southern African Institute of Mining and Metallurgy, vol. 118, no. 8, pp. 753-761.

Cumming-Potvin, D, Wesseloo, J, Jacobsz, SW \& Kearsley, EP 2016b, 'Results from physical models of block caving', Proceedings of MassMin2016, The Australasian Institute of Mining and Metallurgy, Melbourne, pp. 329-340.

Cumming-Potvin, D, Wesseloo, J, Jacobsz, SW \& Kearsley, EP 2018, 'A re-evaluation of the conceptual model of caving mechanics', Proceedings of the Fourth International Symposium on Block and Sublevel Caving, Australian Centre for Geomechanics, Perth, pp. 179-190.

Ewy, RT \& Cook, NGW 1990, 'Deformation and fracture around cylindrical openings in rock II. Initiation, growth and interaction of fractures', International Journal of Rock Mechanics and Mining Sciences \& Geomechanics Abstracts, vol. 27, no. 5, pp. 409-427.

Garza-Cruz, TV \& Pierce, M 2014, 'A 3DEC model for heavily veined massive rock masses', Proceedings of the 48th US Rock Mechanics/Geomechanics Symposium, American Rock Mechanics Association, Alexandria.

Hoek, E 1965, 'The design of a centrifuge for the simulation of gravitational force fields in mine models', Journal of the South African Institute of Mining and Metallurgy, vol. 65, no. 9, pp. 455-487.

Itasca Consulting Group 2016a, 3DEC, computer software, Itasca Consulting Group, Minneapolis, https://www.itascacg.com/software /3dec

Itasca Consulting Group 2016b, FLAC3D, computer software, Itasca Consulting Group, Minneapolis, https://www.itascacg.com/ software/flac3d

Lisjak, A, Figi, D \& Grasselli, G 2014, 'Fracture development around deep underground excavations: Insights from FDEM modelling', Journal of Rock Mechanics and Geotechnical Engineering, vol. 6, no. 6, pp. 493-505.

Meier, T, Rybacki, E, Reinicke, A \& Dresen, G 2013, 'Influence of borehole diameter on the formation of borehole breakouts in black shale', International Journal of Rock Mechanics and Mining Sciences, vol. 62, pp. 74-85.

Stacey, TR 1981, 'A simple extension strain criterion for fracture of brittle rock', International Journal of Rock Mechanics and Mining Sciences \& Geomechanics Abstracts, vol. 18, no. 6, pp. 469-474.

Wesseloo, J \& Stacey, TR 2016, 'A reconsideration of the extension strain criterion for fracture and failure of Rock', Rock Mechanics and Rock Engineering, vol. 49, no. 12, pp. 4667-4679. 
